

\section{The Radiology Impact of Healthcare Errors during Shift Work}

Jason Elliott, Keren Williamson ElliottJ9@cardiff.ac.uk, Williamsonk2@cardiff.ac.uk

School of Healthcare Sciences, Cardiff University; Ty Dewi Sant, Heath Park, Cardiff CF14 4XN

Corresponding Author: Jason Elliott

Keywords: shift work, sleep, staff, error, risk,

\section{ABSTRACT}

Objectives

What are the risks of error as a result of out-of-hours work in health care that could be attributed to Shift Work Disorder; and what safety implications would this have for radiographers working with ionising radiation?

To conduct a literature search of existing studies of Shift Work Disorder

To critically appraise appropriate studies for research rigour

To synthesize and discuss findings in the selected research articles

To investigate the impact of the results in relation to medical imaging and safety

Key Findings

A systematic review of the literature was planned and executed to meet the above objectives. Narrative synthesis was used to describe the heterogeneous findings of the studies appraised.

Conclusion

Four of the five studies selected after critical appraisal suggested a positive correlation of error with increased mental and physical fatigue as a result of shift work or rapid shift rotation. No studies directly investigated medical imaging professionals.

Implication for Practice

Considerations need to be made when optimising shift work for healthcare professionals so as to avoid Shift Work Disorder and consequential error; particularly in the context of ionising radiation. Research into environmental and lifestyle support should be pursued to study its effect as prevention or management. Further direct study with radiographers is recommended 


\section{CONFLICT OF INTEREST}

None.

\section{INTRODUCTION}

In order to meet increasing demand ${ }^{1}$, radiographers worldwide are working more shifts that include time outside of what the UK Working Time Regulations ${ }^{2}$ define as the normal working day, specifically the night hours of 23:00 to 06:00. This often means a closely repeating atypical pattern of day and night shifts, with a substantial portion of a healthcare professional's shifts being undertaken at night. The UK radiography workforce has a $9.1 \%$ vacancy rate $^{3}$, which increases the propensity of working hours during the evening and early morning; in order to maintain a 24/7 delivery of service and bringing staff beyond the limits defined by the Working Time Regulations ${ }^{2}$. It is part of those increasing demands on radiographers, with the advent of extended working days for non-acute care, that switches departments to 12-hour shifts ; covering a greater throughput of routine examinations ${ }^{4}$ and increased referral to specialist imaging modalities such as Computed Tomography (CT) and Magnetic Resonance Imaging (MRI) ${ }^{5}$.

Regularly working evenings and nights can increase the risk of developing a collection of symptoms categorised as Shift Work Disorder $(S W D)^{6}$, diagnosed similarly to Seasonal Affective Disorder (SAD) due to its similarities in symptoms and co-morbidities ${ }^{7}$. SWD is caused by disruption of the body's natural circadian rhythm; a 24-hour cycle of changes in body function according to being awake or asleep. This has been related to recent Nobel Prize awarded research into proteins that are encoded by a gene controlling the biological clock; regulating "critical behaviour" such as metabolism, body temperature and hormone levels ${ }^{8}$. Extended disruption of wakefulness and sleep will break the natural circadian cycle, causing changes within the body's function ${ }^{9}$; resulting in signs of depression, insomnia or excessive sleepiness ${ }^{10}$ and weight gain; with corresponding cardiometabolic consequences ${ }^{11}$.

Additional evidence suggests an increased risk of "attentional failures" and errors as a result of
SWD ${ }^{12}$. This has been alleged to account for a significant degree of accidents and catastrophes including the Chernobyl Power Plant disaster ${ }^{13}$. Studies by Hanecke et al $^{14}$ suggest that later starting times increase the accident risk dramatically towards the end of a shift. Other studies $^{15}$ suggest high fatigue had a serious effect on medical professionals undertaking their work. Hughes and Rogers ${ }^{16}$ suggest manual tasks become more challenging as the night shift goes on.

Reporting of adverse events is important within healthcare, in order to analyse trends and make recommendations to improve practice ${ }^{17}$, including any exposure Much Greater Than Intended (MGTI) according to IRMER $2017^{18}$ Regulation 4(5). This can apply to mis-exposure of a patient who did not require imaging, as well as those where parameters for imaging were in excess of an acceptable national Dose Reference Level $(\mathrm{DRL})^{18}$. The Care Quality Commission (CQC) received 1,219 MGTI reports for England in $2016^{19}, 60 \%$ of which were cited as generalised "operator error". No data exists on the number of incidents outside of the working day 20,21 nor incidents as a result of fatigue.

It is possible to suggest that radiographers may be subject to SWD; and this could lead to grave consequences when working with ionising radiation ${ }^{18}$. If healthcare staff, and therefore radiographers, could be subject to the effects of this circadian disruption and metabolic change, it is key that staff and managers are aware so that these effects can be discussed and managed well.

\section{METHODS}

This investigation follows the formal guidance supplied by Piper $^{22}$ for conducting systematic reviews in a healthcare setting; utilising PICO criteria $^{23}$ and PRISMA group reporting guidance ${ }^{24}$. Due to no results being returned for radiographers, the review criteria expanded to fit other registered healthcare professions considered comparable by work type, shifts and qualifications. 


\section{Population criteria}

INCLUDED - all registered professions undertaking shift work that may fall into the night period of (23:00-06:00)

EXCLUDED - non-registered healthcare / nonshift professions (eg Occupational Therapists), doctors (due to incomparable working practices)

\section{Intervention criteria}

INCLUDED: Healthcare-related shift and out-ofhours work (23:00-06:00), including shift patterns and/or extended days that rotate into these hours

EXCLUDED: studies without work that falls inside these hours (ie day shift, fixed daytime extended work)

\section{Comparison criteria}

INCLUDED: Data comparing other patterns and "daytime" work to shift work

EXCLUDED: Daytime work only; noncomparative studies; studies which do not explore relationships between relevant shift patterns / types

\section{Outcome criteria}

INCLUDED: - Studies investigating error as a result of shift work, including indirect outcomes (ie general shift work studies that include data comparing errors with shift-related outcomes)

EXCLUDED - Studies which do not investigate error as a result of shift work

All quantitative studies available will be included; excluding systematic reviews, conference submissions, opinion pieces and posters. Due to limitations in scope, grey literature will be avoided. Any previous study will be included, with no defined time frame; in English or translated/available in English. Articles which do not provide full text will be discarded.

The scope of investigation for this study consisted of the following databases: MEDLINE, CINAHL plus, PsycINFO, EMBASE, EMCARE, SCOPUS, Web-Of-Science, BIOSIS, with terms included in Table 1.
3,407 studies were retrieved; and underwent primary screening, removing of 1939 duplicates and 1350 irrelevant articles (Appendix A). 88 studies were discarded at the next stage by failing to meet the PICO criteria, for example investigating effects after a shift ${ }^{25}$. Of the ten studies remaining (Appendix B), all were identified as prevalence studies; this reflects the expectation that most studies will observe existing shift patterns in place. All of these utilised a questionnaire in their data collection, with one recording direct quasi experimental data alongside and two using secondary sources to support questionnaire answers. The studies were then critically appraised by two researchers using the JBI Critical Appraisal Checklists ${ }^{26}$ for prevalence study, to assess research quality and rigour. Studies which were appraised as suitable for synthesis were extracted using a detailed data extraction tool26; due to the heterogeneous nature of the data in the remaining 5 studies, a narrative synthesis was conducted using the method described by Popay et $\mathrm{al}^{27}$.

\section{RESULTS}

All extracted studies in Table 2 review issues with impairment of physical or mental health (including sleep deprivation), plus the consequential change in error (either reported or observed) as a result of shift work or rapid shift rotation. Lo et $\mathrm{al}^{30}$ studies the specific risk area of needlestick injuries, whereas the remaining four filtered articles discuss self-reporting of various mistakes (including sharps injuries) during working practice. Barker \& Nussbaum ${ }^{28}$ and Suzuki et $\mathrm{al}^{31}$ directly relate shift work to the results, directly correlating changes to outcomes with the hours and time of day worked whereas the remainder look at the ongoing performance implications of shift change, repeat and switching. The PICO formats all correlate with search strategy criteria, with some variance in control population. All studies observed nurses, who are comparable with the other allied health professions including radiography, however the lack of breadth in participant populations can be considered a limitation for discussion later in the review. The variance in sample sizes reflects the choice of national, local or single-ward populations identified by the researchers, as well as study methods and observations made. 
All studies reviewed sleep, with three reviewing participant health using a range of methods. This may have a more direct approach if the study of shift work disorder was directly implicated, however this systematic review is purely investigating the effects of the shift work itself, not as a secondary complication of fatigue. All but Chang et $\mathrm{al}^{29}$ relied on self-reporting of information on mental and physical fatigue in relation to mental health and sleep quality, and subsequent error or simulated error. As a result of the self-reporting, there may be inherent bias to the outcomes; this is likely to mask the true nature of errors as participants who make mistakes are more likely to under-report in these situations.

Participant uptake was high in all selected studies, with at least $74 \%$ of each sample returning a response. This should improve the quality and validity of any conclusion drawn from the study ${ }^{34}$.

All studies comparably use direct $p$ values to explain the significance of changes between groups of participants. Three studies were specific in their use of comparison tests, usually to compare grouped data responses for shift/no shift ${ }^{30,31}$ or groups of shiftworkers undertaking different consecutive patterns ${ }^{29}$. Additionally, three use SD as a measure of variance within the data to confirm the consistency of response ${ }^{32}$, a fourth ${ }^{28}$ using Cronbach's alpha and derivations to show the same.

While all studies met the criteria of study, and all were deemed appropriate by critical appraisal, they choose to compare different elements to identify the risk or prevalence of error in the sample. Both sleep and mental health detriment can be seen to contribute to Shift Work Disorder and are negatively affected by working unsocial shift patterns or rapidly rotating shifts (that include the night patterns specified in this systematic review).

All studies bar Weaver et $\mathrm{al}^{32}$ suggested a decrease in mental health quality or an increase in sleeplessness because of shift work or increased shift rotation, coupled with a consequential increase in error. This identifies the population presenting with SWD symptoms; however, the methods to conclude this were all different. The methods to identify physical and mental fatigue were dissimilar, differing complex tools were used and none had similar statistical interrogation. All but Weaver et $\mathrm{al}^{32}$ include statistically significant $p$ values on their resultant correlations. This may suggest the last study as an outlier, but this will be considered when reviewing the implications of the 5 study results.

The first clear direct comparison of shift work and error in these studies is from the univariate analysis performed on data in Suzuki et $\mathrm{al}^{31}$; suggesting the odds ratio for error was 2:54 for shift workers in comparison to those who worked a standard day shift. There is also a greater ratio for errors in participant nurses with poor health, insufficient and broken sleep. These have been directly attributed as results of shift work, so can be identified as contributing factors to the fatigue that increases error risk. These had very good confidence intervals, suggesting the scores were of high consistency.

Barker and Nussbaum's ${ }^{28}$ data compared fatigue levels and error, suggesting a correlation. While data was collected on staff undertaking shifts and no shifts, it transpires that these are not directly compared in the study. They are, however, indirectly linked: conclusions state that shift work increases the level of physical and mental fatigue in participants, and separately that these increase the response of error risk according to the Nursing Performance Instrument (NPI) tool used in the study. The NPI scores were correlated to fatigue data with $p$ values, which were statistically significant when discussing measured fatigue on most NPI measured elements of performance. Cronbach's alpha and coefficient were used to prove statistical validity and consistency.

The results from Chang et al's study ${ }^{29}$ suggest that inconsistent out-of-hours shifts cause impairment to "perceptual and motor abilities"; measured by comparing motor skills, sleepiness and anxiety levels (representing overall mental health) in comparison between shift patterns. Motor skill tests recorded speed and errors made, to ascertain the nature of a nurse's consistency in task completion. Outcomes from these measures suggest that decreases in physical and mental health are increasing the risk of error in nursing staff who have rapidly successive changes in working pattern. This in turn affects circadian balance and the ability to perform and care in the hospital environment. This could be applicable in working practices where there is not a continual step change in pattern, for example, on a wideranging rota sporadically covering out-of-hours work in a radiology department ${ }^{35}$. 
The study results from Lo et al ${ }^{30}$ showed a greater odds ratio for both needlestick or other sharps injuries in shift-workers operating above the 40 hour a week range,with significant hikes in risk for those working over 50 hours. Completing this number of hours will include shifts undertaken outside of the normal working day (as scheduled in the Working Time Regulations $^{2}$ ). While this may not be prevalent in countries operating under the Working Time Directive, some exemptions, extensions and working on-call scenarios (particularly in radiography for example) may result in this being more common ${ }^{35}$.

Weaver et $\mathrm{al}^{32}$ show no correlation of working time period and error with a wide SD and a poor $p$ value this would suggest that the significance of data results and their implication are not comparable to the other four studies. The article suggests that the Participant Sleep Quality Index (PSQI) that relates tiredness to error is a generalisation of recent sleep pattern, and not the immediate day before - this may mask the effects of poor sleep from shift rotation on changes in error severity. It also fails to discuss the frequency of error between day and night workers, merely the quality of sleep, therefore its conclusions cannot be contextually compared to the other studies in this review. The Visual Analogue Scale used to record error rates has limitations which may have affected the result, particularly the relative subjectivity of the scale itself ${ }^{37}$. Sample size was smaller than most of the other extracted studies, so the magnitude of the results cannot be considered in the same way. In review, while this study cannot be completely discounted, the corresponding results in the other four studies outweigh it.

\section{DISCUSSION}

Primary findings within literature are increased risk of error due to shift work occurring during night hours, with corresponding SWD type characteristics from sleep and mental health data $28,30,31$. Further increase in error was reported when shifts rapidly rotate between days and nights ${ }^{29}$. First observations conclude that insufficient data exists on registered professions outside nursing and more research should be undertaken on the effects of varied working patterns for any health care professional. Considering the specific safety concerns in departments using ionising radiation ${ }^{38}$, this would be particularly pertinent for diagnostic and therapeutic radiographer populations.
Fast rotating shifts ${ }^{29}$ are of concern due to decreased cognitive ability, with inhibition of motor skills. Within the selected literature there is discussion as to the major error points, often including needle-stick radiographers often train to obtain intravenous access, and this risks consequential infection or extravasation of injected contrast media for patients ${ }^{39}$. Cognitive error could additionally be displayed within radiography as positioning errors or exposure selection, contributing to repeated imaging and greater exposure for the patient, which could be detrimental even if below levels deemed MGTI under IRMER $2017^{18}$. Other risks include errors in patient record management, for example documenting incorrect information regarding sides under examination or even the images being filed electronically under the wrong patient. The consequences of patient selection and data management could be misdiagnosis or delays in reporting ${ }^{40}$, which would cause significant harm for patients, particularly if a NEVER event ${ }^{41}$.

Guidance in the European Working Time Directive ${ }^{42}$ and Working Time Regulations ${ }^{2}$ discourage fast rotation, however this can still be common in smaller radiology units with on-call based night shift ${ }^{35,36}$; classified within a local agreement rather than contracted working hours or as part of an opt-out included in the job role ${ }^{43}$. Increased demand on services is an ongoing issue within the National Health Service, particularly in medical imaging leading to increased expectation on staff covering Computed Tomography imaging and inpatient radiography during the night hours ${ }^{5}$.

The four concurrent studies in this review ${ }^{29,29,31,32}$ suggest that staff self-report more errors after night shifts or shift patterns that overlap into night hours. The data includes negative effects on sleep consistency and mental health reporting, consistent with other nursing studies not included in this review ${ }^{44,45,46}$, Medicine ${ }^{47}$ and studies outside of healthcare $49,50,51$.There were no reportable studies found within selected databases which showed more evidence of the opposing no-correlation conclusion by Weaver et $\mathrm{al}^{32}$. This has relevance to radiographers who operate within departments offering a 24-hour service; and often utilise extended days to improve waiting times for imaging ${ }^{4}$. 
Ongoing personal implications for radiographers making shift-work related errors include the potential for long term personal health issues including metabolic disease ${ }^{10}$, affecting radiology department staffing, recruitment and retention ${ }^{5}$. It is also wise to be aware of the potential for serious and potentially fatal errors within radiology departments ${ }^{51}$. Previously discussed non-drug treatments for Shift Work Disorder, for example bright lights and circadian phase delays, have been proven to have a positive effect in the workplace ${ }^{52}$ and on staff health quality ${ }^{48}$. These may be viable for exploration in the context of environmental changes and good practice guidelines to minimise error risks.

The primary limitation of this study will be considered as publication bias ${ }^{53}$ due to the exclusion of grey literature, studies other than English language and the lack of hand-searching for articles. Additionally, no research was returned other than from nursing. As a result, some generalisation has been made regards comparability of registered healthcare professionals, but this is no substitute for direct investigation.

Studies within this systematic review were all considered as relatively low grade due to their status as non-RCT studies, namely observations or surveys ${ }^{54}$. Therefore, the impact of conclusions drawn will be limited. More research is required with other health professionals - particularly radiographers - to directly assess whether working practices and shift patterns have a similar propensity to Shift Work Disorder and the ongoing consequences, and to ascertain whether any secondary measures can reduce the fatigue and error risk.

Furthermore, some investigation into radiographerspecific health and error rates with respect to day, mixed and night shift patterns should be considered; additionally, studies that could investigate any of the environmental and staffing level impacts previously discussed and recommended would be helpful in choosing the most effective tool for optimising practitioner performance and health status while supporting round-the-clock care delivery.

\section{CONCLUSION}

This systematic review has correlated evidence of increased risk of error in healthcare workers undertaking out-of-hours shift work; and this is of particular concern in the radiology working environment $18,40,51$. While it is impractical for radiographers and other health care professionals to work fixed shifts or operate a system where there is no rotation, some consideration needs to be made regarding optimisation of working practice. This should include:

- Working patterns, with reference made to safe staffing levels ${ }^{55}$ minimising fast rotation between shift type;

- Improving the environment for healthcare staff to work in and considering research into secondary factors such as the light quality or availability within the working area ${ }^{56}$;

- Reviewing support available to healthcare professionals to manage their health and performance in the context of 24-hour service provision ${ }^{56}$, including supported safety procedures to avoid significant mistakes being made. This may include awareness of the Working Time Regulations ${ }^{2}$.

There are additional measures that must be maintained in order to minimise risk for patients undergoing diagnostic imaging with ionising radiation ${ }^{40,51}$. This includes strict systems of work(for example the SCoR's "Pause and check") ${ }^{57}$ to allow for correct identification of patients, accuracy and attention to safety during examination procedures, and quick error reporting as to avoid misdiagnosis and error. Overall, this increased awareness of SWD risks in radiographers should be investigated further to optimise delivery of care and high-quality imaging in the radiography workforce. 


\section{REFERENCES}

1. Maguire D, Dunn P, Mckenna H; How hospital activity in the NHS has changed over time. London: The King's Fund, 2016.

2. Working Time Regulations, 1998 [online] Accessed via http://www.legislation.gov.uk/uksi/1998/1 833/contents/made

3. Society and College of Radiographers; Diagnostic Radiography Workforce Report 2018. London: Society of Radiographers, 2018.

4. Department of Health; Implementing 7 Day working in Imaging Departments: Good Practice Guidance A Report from the National Imaging Clinical Advisory Group. London, Department of Health, 2012.

5. Society and College of Radiographers; The Radiography Workforce: Current Challenges and Changing Needs. London, Society \& College of Radiographers, 2016.

6. Akerstedt T, Wright KP; Sleep Loss and Fatigue in Shift work and Shift Work Disorder. Sleep Medicine Clinical Journal 4, 2009, 257-271.

7. Rosenthal NE, Sack DA, Gillin Jc et al; Seasonal Affective Disorder: A description of the syndrome and preliminary findings with Light Therapy. Archive of General Psychiatry, 1984 41, 72-80.

8. Hall JC, Rosbach M, Young MW; Discoveries of Molecular Mechanisms Controlling the Circadian Rhythm. Nobelförsamlingen 2017 [online]. Accessed via

https://www.nobelprize.org/nobel prizes/ medicine/laureates/2017/advancedmedicineprize2017.pdf (07/02/18).

9. Wright KP, Bogan RK, Wyatt JK; Shift Work and the assessment and management of Shift Work Disorder. Sleep Medicine reviews, $2013 \mathrm{Feb}, 17(1), 41-54$.

10. Drake CL, Roberts $\mathrm{T}$, Richardson $\mathrm{G}$ et al; Shift Work Disorder: Prevalence and Consequences Beyond that of Symptomatic Day Workers. Sleep, December 2004, 27(8), 1453-62.

11. Ruger M, Scheer FA; Effects of circadian disruption on the cardiometabolic system. Review of Endocrine Metabolica Disorders, 2009, 10, 245-260.

12. Lockley SW, Cronin JW, Evans EE et al; Effect of reducing interns' weekly work hours on sleep and attentional failures. $N$ Engl $J$ Med, 2004 Oct 28, 351(18), 1829-37.
13. Mitler MM, Carskadon MA, Czeisler CA et al; Catastrophe, Sleep and Public Policy: Consensus Report. Sleep, 1988 Feb, 11(1), 100-109.

14. Hanecke K, Tiedemann S, Nachreiner F et al; Accident risk as a function of hour at work and time of day as determined from accident data and exposure models for the German working population. Scand J Work Environ Health 1998, 24(3), 43-48.

15. Papp KK, Stoller EP, Sage P et al; The effects of sleep loss and fatigue on residentphysicians; a multi-institutional, mixedmethod study. Acad Med, 2004 May, 79(5), 394-406.

16. Hughes RG, Rogers AE; First, Do No Harm: Are you Tired? AJN, 2004 March, 104(3), 36-38.

17. Shaw $R$, Drever $F$, Hughes $\mathrm{H}$ et al; Adverse events and near miss reporting in the NHS. BMJ, Quality \& Safety, 2005, 14 (4), 279283.

18. Ionising Radiation (Medical Exposure) Regulations, 2017.

19. Care Quality Commission; IR(ME)R Annual Report 2016. Newcastle: Care Quality Commission, 2017.

20. Health and Safety Executive. Managing Shiftwork; Health and Safety Guidance. Merseyside: HSE, 2016.

21. ICRP; Radiological Protection in Medicine. ICRP Publication 105 Ann. ICRP 37(6), 2007.

22. Piper RJ; How to write a systematic literature review: a guide for medical students. London: National Student Association for Medical Research, 2013.

23. Methley AM, Campbell S, Chew-Graham C et al; PICO, PICOS and SPIDER: a comparison study of specificity and sensitivity in three search tools for qualitative systematic literature reviews. BMC Health Serv Res. 2014, 14, 579.

24. Moher D, Liberati A, Tetzlaff J et al; Preferred Reporting Items for Systematic literature reviews and Meta-Analyses: The PRISMA Statement. PLoS Med, 2009, 6(6), e1000097.

25. Asaoka, S, Aritake S, Komada $\mathrm{Y}$ et al; Factors Associated with Shift Work Disorder in Nurses working with Rapid-Rotation schedules in Japan: The Nurses' Sleep health Project. Chronobiology International, May 2013, 30(4), 628-636.

26. Joanna Briggs Institute; Reviewer's Manual, Critical Appraisal Checklists. 2014; Adelaide, 
Joanna Briggs Institute/University of Adelaide

27. Popay J, Roberts H, Sowden A. et al; Guidance on the conduct of narrative synthesis in systematic reviews. ESRC methods programme Version, 1, 92, 2005.

28. Barker LM, Nussbaum MA; Fatigue, Performance and the Work Environment: a survey of registered nurses. Journal of Advanced Nursing, 2010, 67(6), 1370-1382.

29. Chang $\mathrm{YS}, \mathrm{Wu} \mathrm{YH}$, Chung $\mathrm{YH}$ et al; Impairment of perceptual and motor abilities at the end of a night shift is greater in nurses working fast rotating shifts. Sleep Medicine, 2004, 12, 866-869.

30. Lo WY, Chiou ST, Huang $\mathrm{N}$ et al; Long work hours and chronic insomnia are associated with needlestick and sharps injuries in hospital nurses in Taiwan: A National Survey. International Journal of Nursing Studies, 2016, 64, 130-136.

31. Suzuki K, Ohida K, Keneita Y et al; Mental Health Status, Shift Work, and Occupational Accidents among Hospital Nurses in Japan. $J$ Occup Health 2004, 46, 448-454.

32. Weaver AL, Stutzman SE, Supnet $C$ et al; Sleep Quality, but not quantity, is associated with self-percieved minor error rates amongst emergency department nurses. International Emergency Nursing, 2016, 25, 48-52.

33. Polgar S, Thomas SA; Introduction to Research in the Health Sciences, $5^{\text {th }}$ edition. Philadelphia: Elsevier Churchill Livingstone.

34. Chess LE, Gagnier JJ; Applicable or nonapplicable: investigations of clinical heterogeneity in systematic reviews. BMC Medical Res Methodol; 2016 Dec, 16, 19.

35. Hawke FA; Development of a work pattern model to meet the changing service requirements with respect to patient and staff needs in Scottish radiology departments [online]. Scottish Clinical Imaging Network 2011. Accessed via

http://www.scin.scot.nhs.uk/wpcontent/uploads/sites/3/2015/04/Thesisfinal-FH-service-redesign.pdf (15/08/2008).

36. McNicoll AM, Boterill JS; On-call work and health: a review. Environmental Health, 2004, 3, 15.

37. Watson R, Atkinson I, Egerton P; Successful Statistics for Nursing and Healthcare. Hampshire: Palgrave Macmillan, 2006.

38. Picano E; Informed Consent and communication of risk from radiological and nuclear medicine examinations: how to escape from a communication inferno. BMJ, 2004 Oct 9, 329(7470), 849-51.

39. Bezhadi AH, Farooq Z, Newhouse JH et al; MRI \& CT Contrast Extravasation. Medicine (Baltimore), 2018 Mar, 97(9), e0055.

40. Denham G, Page N, 2017; Recommendations for Radiographers and radiation therapists drawn from an analysis of errors on Australian Radiation Incident Registers. Journal of Medical Radiation Sciences, 2017 Sep, 64(3), 165-171.

41. Patient Safety Wales, 2013; Never Events. [online] Accessed via http://www.patientsafety.wales.nhs.uk/neverevents (14/03/2018).

42. European Working Time Directive, 2003 [online]. Accessed via http://eurlex.europa.eu/LexUriServ/LexUriServ.do ?uri=CELEX:32003L0088:EN:NOT $(25 / 02 / 18)$

43. NHS Employers; NHS Terms and Conditions of Service Handbook, amendment 39 [online]. NHS Employers 2018. Accessed via http://www.nhsemployers.org/employers handbook/afc tc of service handbook fb.pdf $(29 / 07 / 18)$

44. Caruso CC; Negative Impacts of Shift work and long hours. Rehabil Nurs, 2014 Jan-Feb, 39(1), 16-25.

45. Johnson AL, Jung L, Song $Y$ et al; Sleep deprivation and error in nurses who work the night shift. J Nurs Adm, 2014 Jan, 44(1), 1722.

46. Rheaume A, Mullen J; The impact of long hours and shift work on cognitive errors in nurses. J Nurs Manag 2018, 26, 26-32.

47. Jackson EJ, Moreton A; Safety during night shifts: a cross-sectional survey of Junior Doctors' preparation and practice. BMJ Open, 2013, 3, e003567.

48. Arendt J; Shift Work: Coping with the Biological Clock. Occupational Medicine, Jan 2010, 60(1), 10-20.

49. Kazemi $R$, Haidarimoghadam $R$, Motamedzadeh $\mathrm{M}$ et al; The effects of shift work on cognitive performance, sleep quality and sleepiness among petrochemical control room operators. Journal of Circadian rhythms, 2016, 14(1), 1-8.

50. Ryu J, Jung-Choi K, Choi KH et al; Associations of Shift Work with Work-related injury among electronics factory workers in South Korea. Int J Environ Res Public Health, 2017 Nov, 14(11), 1429.

51. Serranheira F, Proenca J; X-ray active failiures: behind radiographer's errors. In: 
Albolino S, Bagnara S, Bellandi et al; Healthcare Systems Ergonomics and Patient Safety. Boca Raton: CRC Press, 2011.

52. Viola AU, James LM, Schlangen LJ et al; Blue-enriched white light in the workplace improves self-reported alertness, performance and sleep quality. Scandinavian Journal of Work Environmental Health; 2008, August, 34(4), 297-306.

53. Ahmed I; Assessment of publication bias, selection bias, and unavailable data in metaanalyses using individual participant data: a database survey. BMJ 2012, 344, d7762.

54. Thoma A, Eaves FF; A Brief History of Evidence Based Medicine. Aesthetic Surgery Journal 2015, 35(8), NP261-NP263
55. Society and College of Radiographers; Principles of Safe Staffing for Radiography Leaders. London: Society \& College of Radiographers, 2015.

56. Crowley SJ, Lee C, Tseng CY et al; Combinations of Bright Light, Scheduled Dark, Sunglasses and melatonin to facilitate circadian entrainment to night work. J Biol Rhythms, Dec 2003, 18(6), 513-23.

57. Society and College of Radiographers; Have You Paused and Checked? 2016, [online] accessed via

https://www.sor.org/learning/documentlibrary/have-you-paused-and-checked-irmer $(25 / 01 / 18)$ 
PRISMA 2009 Flow Diagram

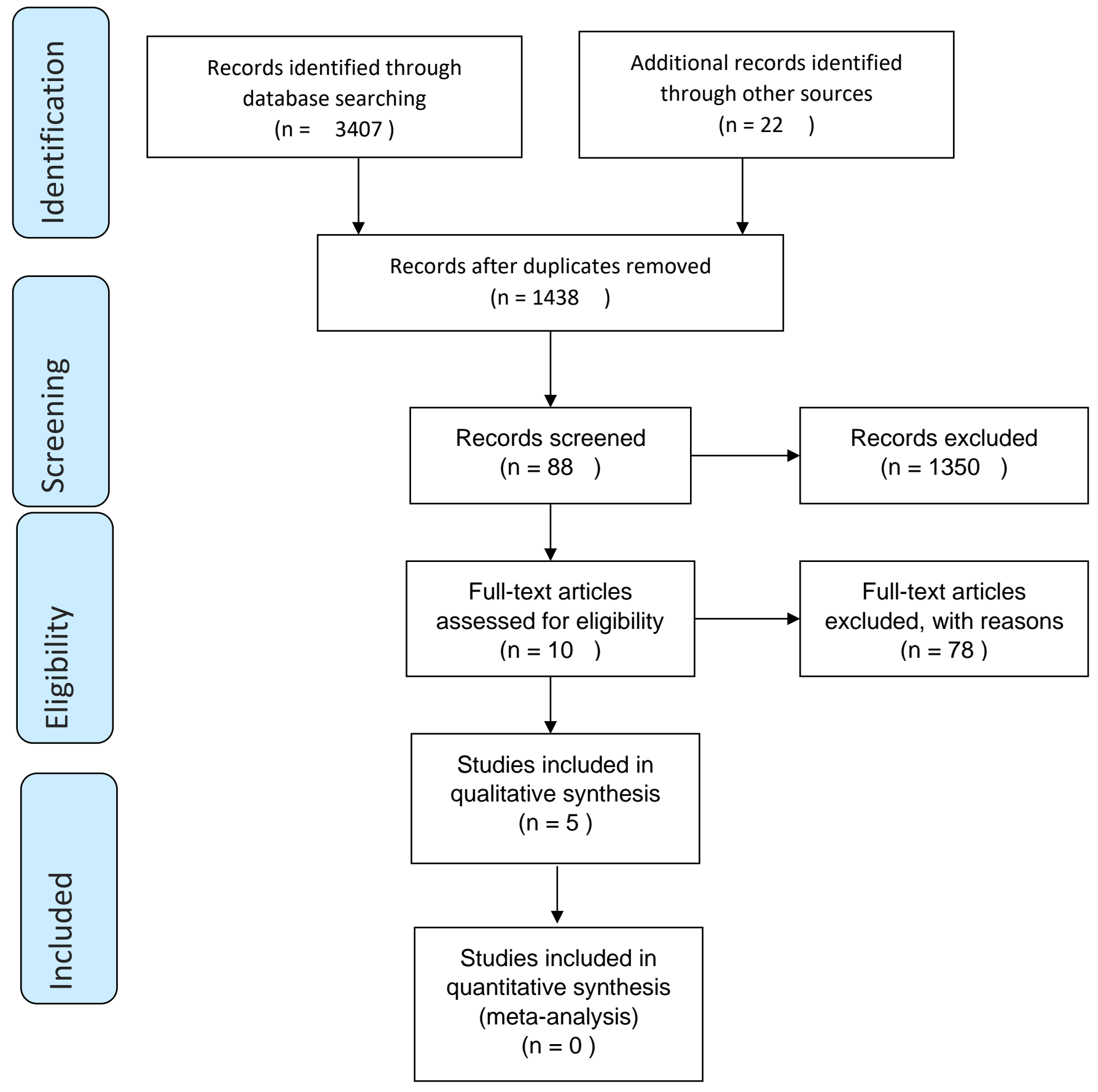




\section{APPENDIX B: SHORTLISTED STUDIES FOR APPRAISAL}

- Barker LM, Nussbaum MA; Fatigue, Performance and the Work Environment: a survey of registered nurses. Journal of Advanced Nursing, 2010, 67(6), 1370-1382.

- Chang YS, Wu YH, Chung YH et al; Impairment of perceptual and motor abilities at the end of a night shift is greater in nurses working fast rotating shifts. Sleep Medicine, 2004, 12, 866-869.

- Dorrian J, Lamond N, van den Heuvel C et al; A Pilot Study of the safety implications of Australian Nurses' sleep and work hours. Chronobiology International, 2006, 23(6), 1149-1163.

- Gold DR, Gogacz S, Bock N et al; Rotating Shift Work, Sleep, and Accidents related to Sleepiness in Hospital Nurses. Am J Public Health, 1992, 82, 1011-1014.

- Johnson AL, Weaver MT, Jung L et al; Sleep Deprivation and Error in Nurses that work the Night Shift. Journal of Nursing Administration, 2014, 44(1), 17-22.

- Lo WY, Chiou ST, Huang N et al; Long work hours and chronic insomnia are associated with needlestick and sharps injuries in hospital nurses in Taiwan: A National Survey. International Journal of Nursing Studies, 2016, 64, 130-136.

- Merchaoui I, Chaari N, Bouhlel M et al; Influence of Shift work on Manual Dexterity and Reaction Time in Tunisian Nurses. Recent Patents on Inflammation \& Allergy Drug Discovery, 2017, 11, 129-135.

- Saleh AM, Awadalla NJ, El-Masri YM et al; Impacts of nurses' circadian rhythm disorders, fatigue and depression on medication administration errors. Egyptian Journal of Chest Diseases and Tuberculosis, 2014, 63, 145-153.

- Suzuki K, Ohida K, Keneita Y et al; Mental Health Status, Shift Work, and Occupational Accidents among Hospital Nurses in Japan. J Occup Health 2004, 46, 448-454.

- Weaver AL, Stutzman SE, Supnet C et al; Sleep Quality, but not quantity, is associated with selfpercieved minor error rates amongst emergency department nurses. International Emergency Nursing, 2016, 25, 48-52. 
Table 1 - Search Terms

\begin{tabular}{|c|c|c|c|}
\hline Population & Workload & Location & Outcome \\
\hline Nurse $^{\star}$ & Night $^{*}$ & Hospital $^{*}$ & Job Performance \\
\hline Radiographer* & Shiftwork* & Ward* & Patient safety \\
\hline Midwife* & Out-of-hours & Department $^{*}$ & Adverse Outcome* \\
\hline Midwives & "out-of-hours" & Acute Setting* & \\
\hline Operating Department Practitioner* & Extended hours & Health Facility & \\
\hline Paramedic* & & & \\
\hline Physiotherapist* & \multicolumn{3}{|c|}{ Key: ${ }^{*}=$ wildcard allows for search of any terms that include these words } \\
\hline
\end{tabular}

Table 2 - Studies selected for Narrative Synthesis

\begin{tabular}{|c|c|c|c|c|c|}
\hline Ref & Author & Year & Title & Journal & Details \\
\hline 28 & Barker \& Nussbaum & 2010 & $\begin{array}{l}\text { Fatigue, performance and the work environment: a study of } \\
\text { registered nurses }\end{array}$ & Journal of Advanced Nursing & $\begin{array}{l}\text { Correlates Fatigue levels \& Performance. Nurses across multiple US } \\
\text { Hospitals completed Health Status questionnaires \& error incidence. } \\
\text { Comparison between Shift and no-shift (daytime) }\end{array}$ \\
\hline 29 & Chang et al & 2004 & $\begin{array}{l}\text { Impairment of perceptual and motor abilities at the end of a } \\
\text { night shift is grater in nurses working fast rotating shifts }\end{array}$ & Sleep Medicine & $\begin{array}{l}\text { Compares performance from varying day-night shift rotation. Nurses } \\
\text { on specific wards in Taiwan completed questionnaires and undertook } \\
\text { tasks to measure cognition and perception. Comparison made } \\
\text { between fast and slow rotating shift patterns }\end{array}$ \\
\hline 30 & Lo et al & 2016 & $\begin{array}{l}\text { Long work hours and chronic insomnia are associated with } \\
\text { needlestick and sharps injuries among hospital nurses in } \\
\text { Taiwan: A national survey }\end{array}$ & $\begin{array}{l}\text { International Journal of } \\
\text { Nursing Studies }\end{array}$ & $\begin{array}{l}\text { Examines whether long work hours and chronic insomnia are } \\
\text { associated with needlestick and sharps injuries among hospital } \\
\text { nurses in Taiwan. Nurses across whole of Taiwan completed } \\
\text { needlestick reporting and sleep questionnaires. Comparison by shift } \\
\text { pattern/rotation }\end{array}$ \\
\hline 31 & Suzuki et al & 2004 & $\begin{array}{l}\text { Mental Health Status, Shift Work, and Occupational Accidents } \\
\text { amongst Hospital Nurses in Japan }\end{array}$ & Journal of Occupational Health & $\begin{array}{l}\text { Examines the Mental Health effects of shift \& Error. Nurses in } \\
\text { multiple hospitals across Japan completed Health/Sleep } \\
\text { Questionnaire and indicated error incidence. Comparison made } \\
\text { between Shift workers and non-shift (daytime) }\end{array}$ \\
\hline 32 & Weaver et al & 2015 & $\begin{array}{l}\text { Sleep quality, but not quantity, is associated with self- } \\
\text { perceived minor error rates among emergency department } \\
\text { nurses }\end{array}$ & $\begin{array}{l}\text { International Emergency } \\
\text { Nursing }\end{array}$ & $\begin{array}{l}\text { Investigating the effects of fatigue on emergency nurse error. ED } \\
\text { Nurses across one state in US completed health questionnaires and } \\
\text { submitted sleep data from smartwatches. Comparison made between } \\
\text { fatigued staff and non-fatigued staff }\end{array}$ \\
\hline
\end{tabular}

